\title{
Preservation of the nerves to the frontalis muscle during pterional craniotomy
}

\author{
Tomas Poblete, MD, Xiaochun Jiang, MD, Noritaka Komune, MD, PhD, Ken Matsushima, MD, and \\ Albert L. Rhoton Jr., MD \\ Department of Neurological Surgery, University of Florida, Gainesville, Florida
}

\begin{abstract}
OBJECT There continues to be confusion over how best to preserve the branches of the facial nerve to the frontalis muscle when elevating a frontotemporal (pterional) scalp flap.The object of this study was to examine the full course of the branches of the facial nerve that must be preserved to maintain innervation of the frontalis muscle during elevation of a frontotemporal scalp flap.
\end{abstract}

METHODS Dissection was performed to follow the temporal branches of facial nerves along their course in 5 adult, cadaveric heads ( $n=10$ extracranial facial nerves).

RESULTS Preserving the nerves to the frontalis muscle requires an understanding of the course of the nerves in 3 areas. The first area is on the outer surface of the temporalis muscle lateral to the superior temporal line (STL) where the interfascial or subfascial approaches are applied, the second is in the area medial to the STL where subpericranial dissection is needed, and the third is along the STL. Preserving the nerves crossing the STL requires an understanding of the complex fascial relationships at this line. It is important to preserve the nerves crossing the lateral and medial parts of the exposure, and the continuity of the nerves as they pass across the STL. Prior descriptions have focused largely on the area superficial to the temporalis muscle lateral to the STL.

CONCLUSIONS Using the interfascial-subpericranial flap and the subfascial-subpericranial flap avoids opening the layer of loose areolar tissue between the temporal fascia and galea in the area lateral to the STL and between the galea and frontal pericranium in the area medial to the STL. It also preserves the continuity of the nerve crossing the STL. This technique allows for the preservation of the nerves to the frontalis muscle along their entire trajectory, from the uppermost part of the parotid gland to the frontalis muscle.

http://thejns.org/doi/abs/10.3171/2014.10.JNS142061

KEY WORDS facial nerve; temporal branch; interfascial; pterional craniotomy; anatomy

$\mathrm{T}$ HE surgical approaches to the frontotemporal region require consideration of preserving the temporal branches of the facial nerve, because damage to them may result in paralysis of the frontalis, orbicularis oculi, and corrugator supercilii muscles. ${ }^{3,17}$ In 1984, Yaşargil et al. ${ }^{16}$ described the interfascial flap technique to protect the facial nerve while allowing maximum retraction of the temporalis muscle during the pterional approach. Interfascial exposure has reduced the incidence of frontalis muscle palsy; however, researchers have been unable to reproduce preservation of the facial nerve..$^{3-5}$ At courses for trainees, we and others ${ }^{11}$ have noted there is still confusion about the best method of dissection to preserve the branches of the facial nerve to the frontalis muscle.
In this study, we examined the course of the temporal branches of the facial nerve from the stylomastoid foramen and parotid gland and across the superior temporal line (STL) to the frontalis and orbicularis oculi muscles (Figs. 1 and 2). The full course of these branches, which have been referred to as temporal, frontal, frontotemporal, or temporofrontal branches, has not been described in detail. $2,8,11,14$ Some papers have focused on dissection superficial to the temporalis muscle over the lateral part of the exposure, failing to emphasize the importance of maintaining the continuity of the temporal branches of the facial nerve at and after they cross the STL to reach the outer surface of the frontal pericranium. This has detracted from an understanding of the full course of the facial

ABBREVIATIONS STA = superficial temporal artery; STL = superior temporal line.

SUBMITTED September 4, 2014. ACCEPTED October 7, 2014.

INCLUDE WHEN CITING Published online April 3, 2015; DOI: 10.3171/2014.10.JNS142061.

DISCLOSURE The authors report no conflict of interest concerning the materials or methods used in this study or the findings specified in this paper. Funding was received from the University of Florida Foundation. 


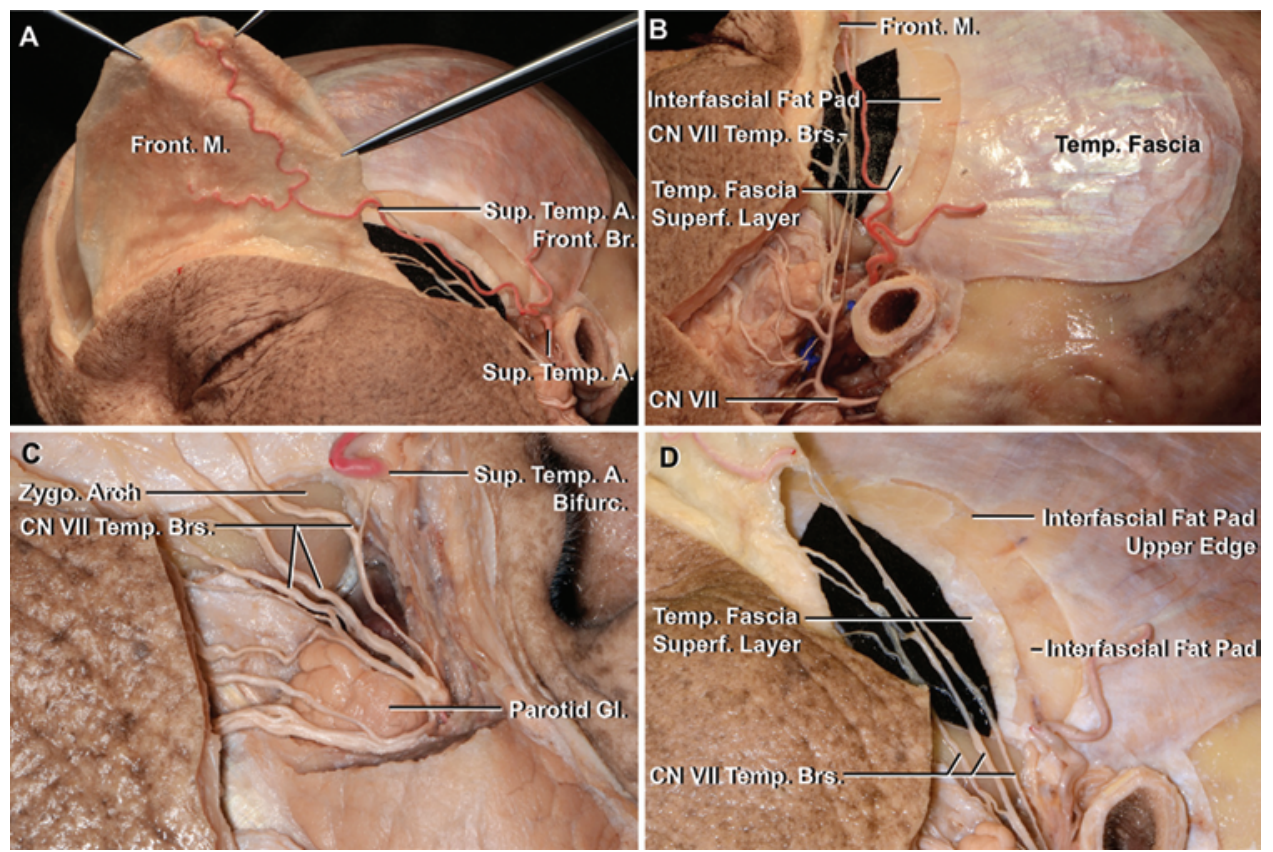

FIG. 1. Photographs of the dissection of the left frontotemporal region of cadaveric human head. A: The frontalis muscle and the galea in which the muscle is embedded have been elevated while preserving the full course of the temporal branches of cranial nerve $(\mathrm{CN}) \mathrm{VII}$ from the stylomastoid foramen to the muscle. The nerves course in the loose areolar tissue on the outer surface of the superficial layer of temporal fascia and the frontal pericranium, and cross the STL to pass under the lateral margin of the frontalis muscle to innervate it from the deep side. Some dark material has been placed under the nerves to highlight their course. B: Lateral view showing the full course of the nerves from the stylomastoid foramen, through the parotid gland, and across the zygomatic arch, superficial layer of temporal fascia, STL, and outer surface of the frontal pericranium to reach the frontalis muscle. C: Enlarged view. There are typically 3 temporal branches of $\mathrm{CN} \mathrm{VII-anterior,} \mathrm{middle,} \mathrm{and} \mathrm{posterior-that} \mathrm{cross} \mathrm{the} \mathrm{zygomatic} \mathrm{arch}$ to innervate the orbicularis, frontalis, and corrugator muscles. D: A window created in the superficial layer of temporal fascia to expose the interfascial fat pad. $\mathrm{A} .=$ artery; Bifurc. = bifurcation; $\mathrm{Br}(\mathrm{s})$. = branch(es); Front. = frontal or frontalis; $\mathrm{Gl} .=$ gland; $\mathrm{M} .=$ muscle; Sup. = superior; Superf. = superficial; Temp. = temporal; Zygo. = zygomatic.

nerve. ${ }^{711}$ Preserving the nerves to the frontalis muscle also requires an understanding of the complex fascial relationships at the STL..$^{2-4,6,7,11,14,18}$

\section{Methods}

The temporal branches of the facial nerve were examined along their course from the stylomastoid foramen and parotid region to the orbicularis oculi and frontalis muscles in 5 formalin-fixed cadaveric heads $(\mathrm{n}=10$ facial nerves). A caliper accurate to within $0.02 \mathrm{~mm}$ (Draper) was used for measurements. Two additional cadaveric specimens were used to demonstrate the dissection techniques that best preserve the anatomical integrity of the temporal branches of the facial nerve.

\section{Results}

Our findings are divided into sections dealing with the nerves in the following 3 areas of the frontotemporal region: 1) on the outer surface of the temporalis muscle lateral to the STL where the interfascial or subfascial approaches are applied, 2) in the area medial to the STL where subpericranial dissection is needed, and 3) along the STL. The fat pads in the temporal region are discussed together.

The superficial and deep layers of temporal fascia, the temporalis muscle, and the periosteum underlying the temporalis muscle extend from laterally and attach to the STL, and the frontal pericranium extends from medially to attach to the STL (Fig. 3). It is important to preserve the nerves crossing the STL between the lateral and medial parts of the exposure. Two commonly used techniques, described as the interfascial and subfascial approaches, have been used to reduce injury to the nerves to the frontalis muscle; however, the terms "interfascial" and "subfascial" apply only to the part of the exposure over the temporalis muscle lateral to the STL. 7,16 To highlight the importance of the approaches medial and lateral to the STL, we refer to them as the interfascial-subpericranial or subfascialsubpericranial approaches.

\section{Layers of the Scalp \\ Layers Lateral to the STL}

The soft-tissue layers of the scalp, from superficial to deep, over the part of the frontotemporal flap lateral to the STL consist of the skin and subcutaneous tissue, the galea, loose areolar tissue, temporal fascia and associated fat pads, the temporalis muscle, and the periosteum (Fig. 3). ${ }^{8,11}$ The periosteal layer that binds the temporalis muscle to the skull has also been referred to as the pericranium, but we use the term periosteum to describe that layer to distinguish it from the pericranium medial to the STL. The frontalis muscle is embedded in the galeal layer (Fig. 1A). The nerves to the frontalis muscle course in the loose 

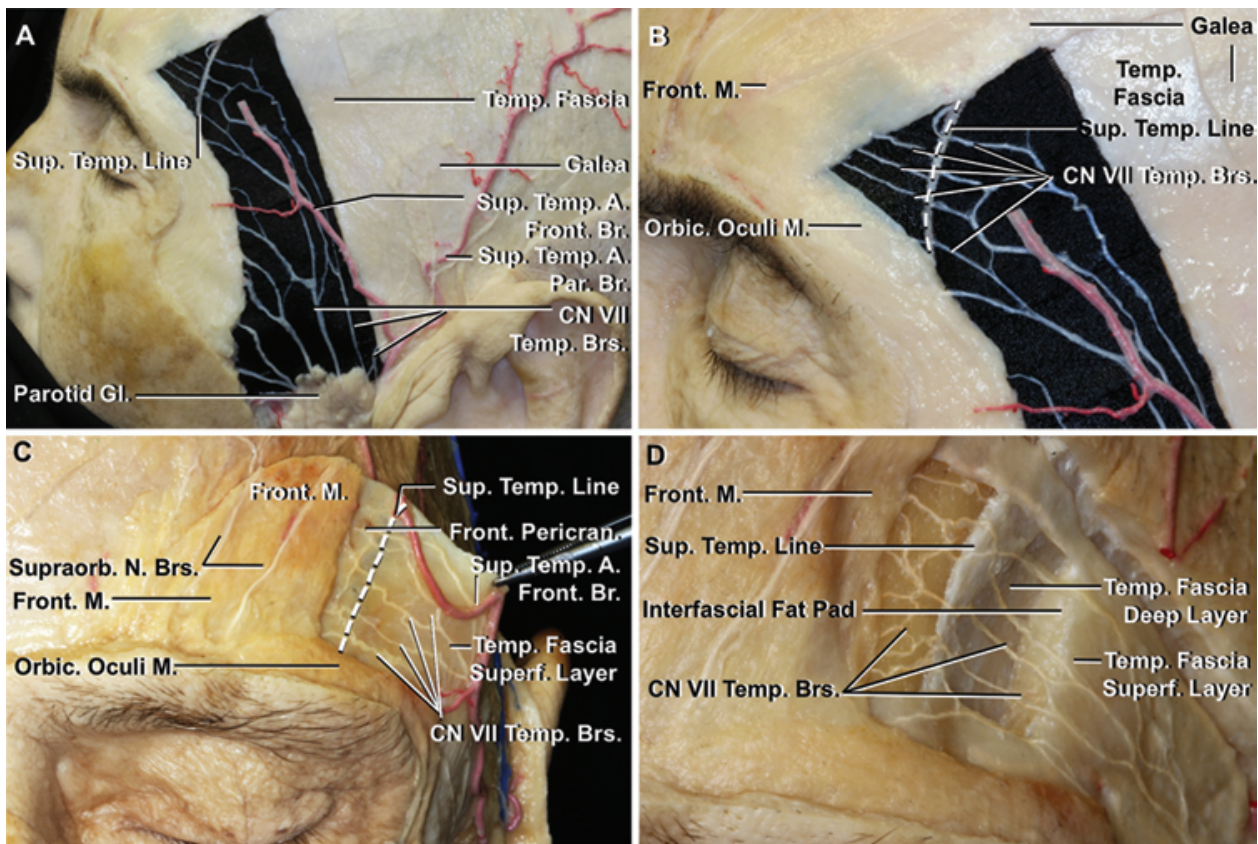

FIG. 2. Close-up photographs of the dissection of left frontotemporal region of cadaveric human head. A: The skin, subcutaneous tissues, and galea have been removed to expose the facial branches coursing in the loose areolar tissue on the outer surface of the temporal fascia. The trunks of the facial nerve exiting the upper edge of the parotid gland and crossing the outer surface of the temporal fascia and frontal pericranium are exposed. Black material has been placed deep to the small branches of CN VII to highlight their course in the loose areolar tissue on the outer surface of the temporal fascia. There are commonly 3 temporal trunks - anterior, middle, and posterior-at the level of the zygomatic arch. The anterior and middle temporal branches supply the orbicularis and frontalis muscles. The posterior temporal branch commonly supplies the auricularis muscle. B: Enlarged view showing the 3 temporal branches at the level of the parotid gland have divided into 5 tiny branches as they cross the STL (interrupted line). C and D: Stepwise dissection shows the relationship of the nerves to the frontalis muscle to the superficial layer of temporal fascia, frontal pericranium, lateral edge of the frontalis muscle, and STL. C: Anterior view. The skin and subcutaneous tissues and galea have been removed to expose the nerves to the frontalis muscle coursing in the loose areolar layer on the outer surface of the superficial layer of temporal fascia and frontal pericranium. The nerves supplying the frontalis muscle pass below the lateral edge of the anterior part of the muscle. The superficial layer of temporal fascia attaches at the STL (interrupted line) and blends medially into the frontal pericranium. The supraorbital branches of CN V ascend on the outer surface of the frontalis muscle. D: A window in the fascial layers adjoining the STL has been completed by removing the superficial layer of temporal fascia and the frontal pericranium to show the nerves to the frontalis muscle crossing the STL. The temporal fascia at and below the level of the upper edge of the interfascial fat pad splits into deep and superficial layers that enclose it. N. = nerve; Orbic. = orbicularis; Par. = parietal; Pericran. = pericranium; Supraorb. = supraorbital. See Fig. 1 for definitions of other abbreviations.

areolar tissue between the temporal fascia and the galea lateral to the STL and between the galea and pericranium medial to the STL, and pass under the lateral edge of the anterior part of the frontalis muscle to innervate it from its deep surface. The nerves pass under the muscle within 2 $\mathrm{cm}$ above the orbital rim.

The skin contains the hair follicles and numerous sebaceous glands. The subcutaneous tissue is a thin layer of fat and fibrous tissue that lies just beneath the skin. ${ }^{8,11}$ The galea, in which the frontalis muscle is embedded, is the broad, musculofibrous layer that extends from the external occipital protuberance and supreme nuchal lines to the eyebrow. The galea merges posteriorly and laterally with the occipital belly of the occipitofrontalis muscle and auricular muscles. ${ }^{1,8,11,14}$ The galea over the temporalis muscle has also been referred to as the frontotemporal fascia. ${ }^{8}$

The loose areolar tissue provides an easy plane of dissection between the galea and the pericranium and outer surface of the temporal fascia. ${ }^{1,8}$ It is through this layer that the nerves to the frontalis muscle pass. It is more difficult to dissect this layer above the zygomatic arch and lateral to the STL, where it gets fattier and where fibrous adhesions bind the galea with the underlying temporal fascia. ${ }^{14}$ The galea on the medial side of the STL differs from that lateral to the STL, because the frontalis muscle is embedded in the 6-7 cm of the galea above the supraorbital rim.

The temporalis muscle, along with its fascia on the outer surface and its periosteum on its deep surface, differentiates the lateral from the medial part of the flap. This muscle and related fascial layers are present only over part of the flap lateral to the STL. ${ }^{8,11}$ The temporal fascia invests and is bounded to the outer surface of the temporalis muscle. ${ }^{1,8,11}$ It is continuous medial to the STL with the frontal pericranium; inferiorly, it attaches to the zygomatic arch. ${ }^{8,11}$ Approximately $4 \mathrm{~cm}$ above the lateral orbital rim, the temporal fascia splits into superficial and deep layers, between which sits the interfascial fat pad..$^{7,8,15}$ The fibrous layer attached to the skull differs in the area medial and lateral to the STL. Lateral to the STL, its outer surface is bound to the deep surface of the temporalis muscle, and its deep or periosteal surface attaches to the bone. Medial to the STL, the outer surface of the pericranium faces the 

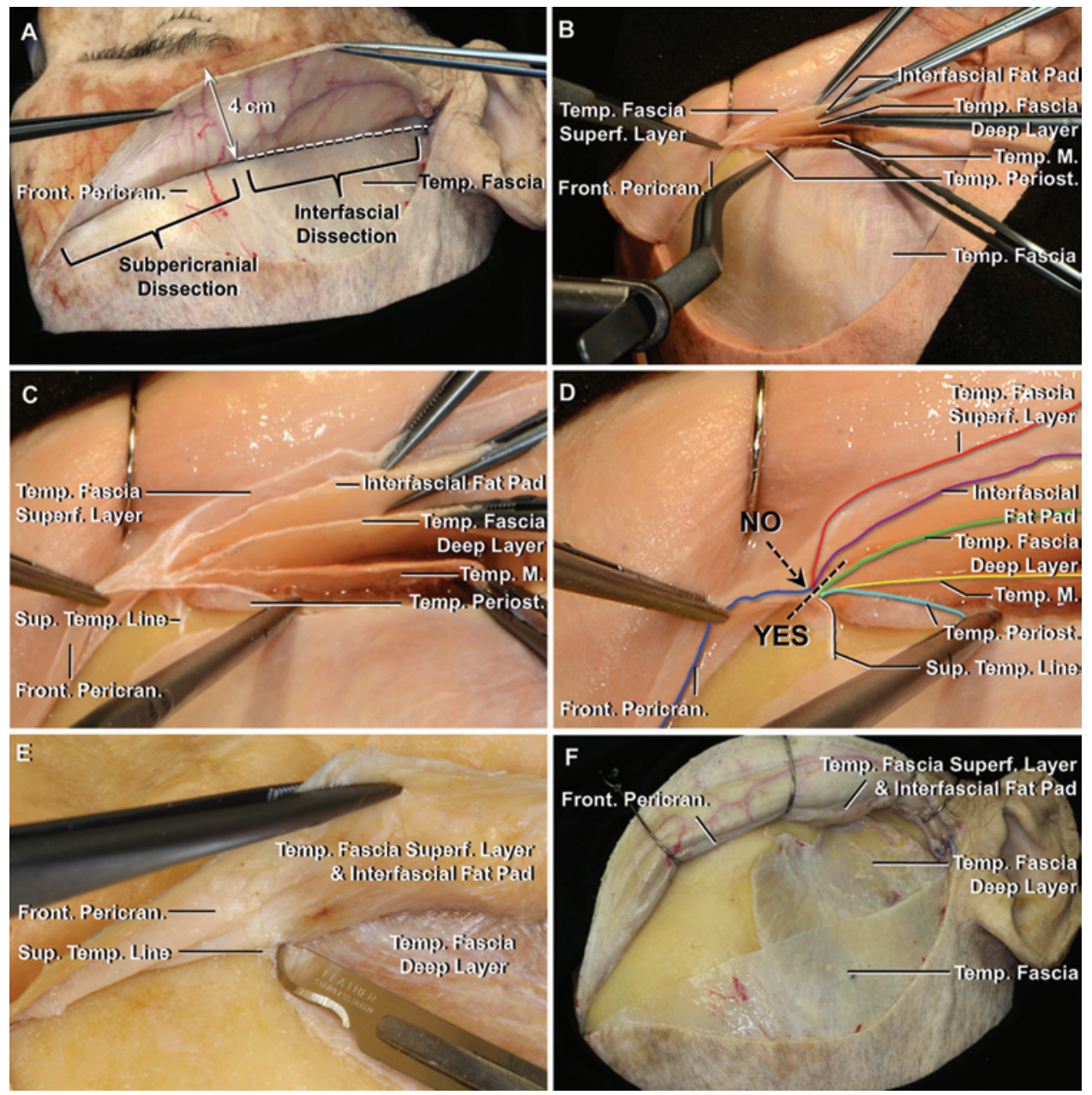

FIG. 3. Photographs of the right frontotemporal exposure. Stepwise dissection shows the layers of the scalp attached along the STL. A: The interfascial dissection starts approximately $4 \mathrm{~cm}$ above the lateral orbital rim at the upper edge of the interfascial fat pad (interrupted line). B: The layers converging on or attaching from the lateral side to the STL are, from superficial to deep, the superficial and deep layers of temporal fascia enclosing the interfascial fat pad, the temporalis muscle, and the temporal periosteum. The frontal pericranium is the only layer medial and attached to the STL. In the interfascial-subpericranial approach, to fold the scalp flap downward over the orbital rim, the superficial layer of temporal fascia lateral to the STL and the pericranium medial to the line must be separated from their attachment to the STL while maintaining the continuity of these 2 layers with the nerves on their outer surface across the STL. The incision in the superficial layer of temporal fascia has been extended across the frontal pericranium $4 \mathrm{~cm}$ above the lateral orbital rim. C: Enlarged view of B. D: Schematic view of the layers attached along the STL: superficial (red) and deep layer (green) of temporal fascia, interfascial fat pad (purple), temporalis muscle (yellow), temporal periosteum (light blue), and frontal pericranium (dark blue). There is a tendency to want to cut vertically across the junction of the frontal pericranium and superficial layer of temporal fascia at the STL, but an incision directed vertically across this junction at the STL will cut the nerves to the frontalis muscle. The cut separating the scalp flap from the STL should extend parallel to the outer surface of the skull while maintaining the continuity between the frontal pericranium medially and the superficial layer of temporal fascia laterally. E: The junction of the superficial layer of temporal fascia and frontal pericranium is being separated from the STL using a cut parallel to the surface of the skull. F: The interfascial-subpericranial flap has been folded downward. Periost. = periosteum; Temp. = temporal or temporalis. See Figs. 1 and 2 for definitions of other abbreviations.

loose areolar tissue; its deep surface attaches directly to the surface of the skull and is referred to as the frontal pericranium. ${ }^{8}$

\section{Layers Medial to the STL}

The layers of the scalp medial to the STL are the skin and subcutaneous tissues, the galea, loose areolar tissue, and the frontal pericranium. Depending on the patient's craniofacial morphology, some of the fat pad in the loose areolar tissue, referred to as the subgaleal fat pad, may extend medial to the STL.

\section{Superior Temporal Line}

The superficial and deep layers of temporal fascia, the temporalis muscle, and the periosteum on the deep side of the temporalis muscle attach from the lateral side to the STL, and the frontal pericranium attaches to the STL from the medial side (Fig. 3B and C). Because the superficial and deep layers of temporal fascia attach to each other and to the frontal pericranium at the STL, it will not be possible to fold the interfascial-subpericranial or subfascial-subpericranial flaps forward over the orbital rim if they remain attached along the STL. Separating the 
temporal fascia from the STL while maintaining the continuity of the nerves crossing the STL between the outer surface of the temporal fascia and the frontal pericranium is needed to allow the flap to be folded forward (Figs. 3, $4 \mathrm{C}$ and $\mathrm{D}$, and $5 \mathrm{~B}$ ).

\section{Fat Pads}

There are 3 fat pads in the temporal region: ${ }^{3,7}$ the sub- gleal fat pad, the interfascial fat pad, and the deep fat pad. The presence and size of these fat pads depend on the individual's craniofacial morphology. ${ }^{8}$ 1) The subgaleal fat pad lies in the loose areolar tissue between the galea and the superficial layer of the temporal fascia and frontal pericranium. ${ }^{8}$ It is through the loose areolar tissue and this fat pad that the facial nerve branches pass. 2) The interfascial fat pad lies between the superficial and deep layers
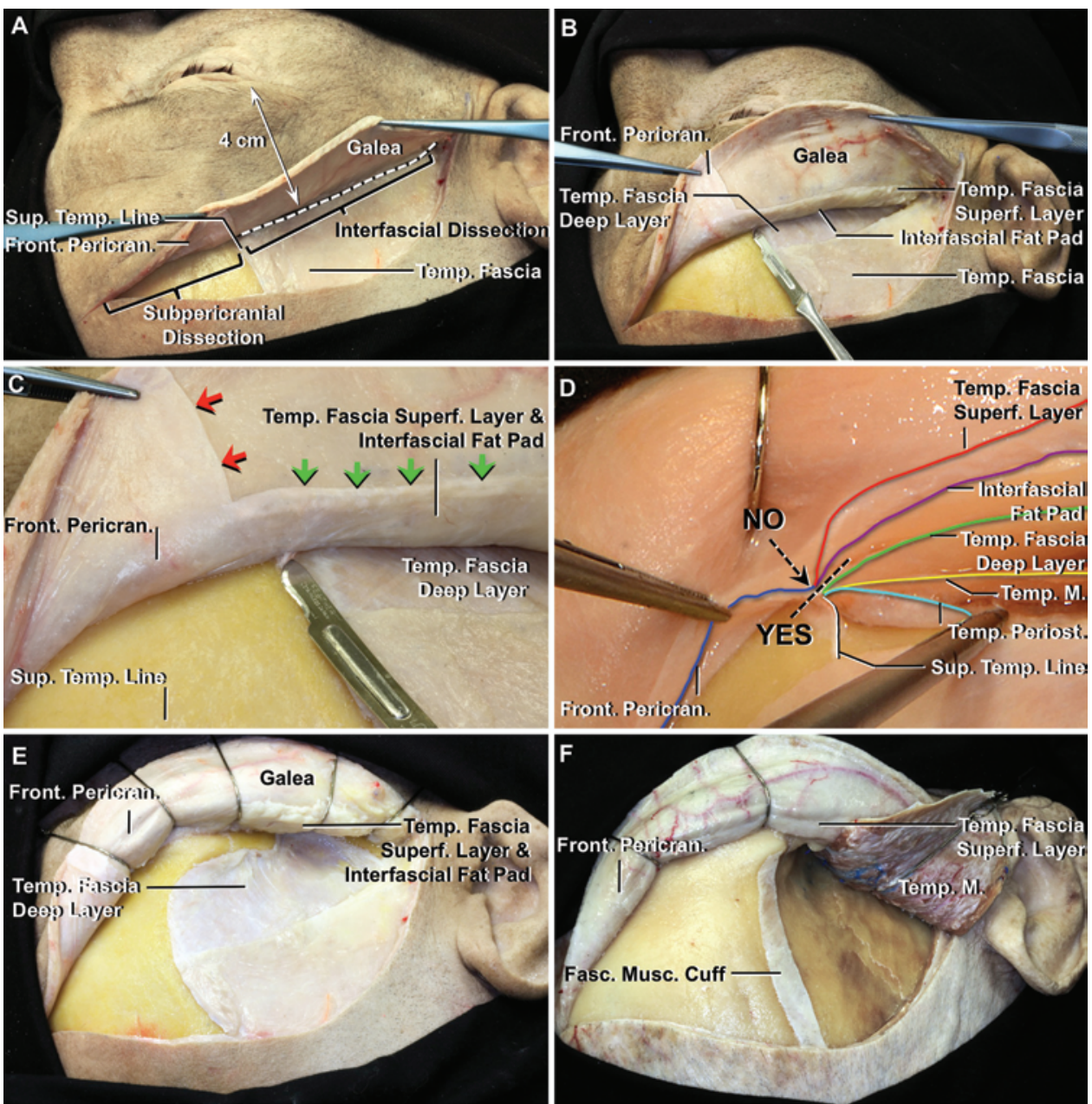

FIG. 4. Photographs of the right interfascial-subpericranial flap and underlying structures. A: The scalp incision rises perpendicular to the zygomatic arch and curves anteriorly toward the midline just behind the hairline. The upper part of the incision lateral to the STL is directed between the galea and temporal fascia. Approximately $4 \mathrm{~cm}$ above the lateral orbital rim (interrupted line), the plane of dissection lateral to the STL is deepened using an incision through the superficial layer of the temporal fascia at the upper edge of the interfascial fat pad. This enables the superficial layer of temporal fascia with the facial nerve branches on its outer surface and the adjacent frontal pericranium medial to the STL to be elevated with the scalp flap. B: The galea is separated from the upper part of the outer surface of the temporal fascia, but the galea and superficial layer of temporal fascia below the interfascial incision are not separated. The upper edge of the interfascial fat pad can be seen through the temporal fascia. In the area medial to the STL, the frontal pericranium is separated from the bone. The continuity between the superficial layer of temporal fascia and the frontal pericranium is preserved to maintain the continuity of the nerves to the frontalis muscle, which cross the outer surface of these structures and the STL. C: To fold the frontotemporal scalp flap forward, the deep side of the junction of the superficial layer of temporal fascia lateral to the STL and the frontal pericranium medial to the STL must be separated from their attachments to the STL while the continuity between the frontal pericranium and superficial layer of temporal fascia is maintained. The green arrows show the cut in the superficial layer of temporal fascia at the upper edge of the interfascial fat pad, and the red arrows show the cut along the frontal pericranium. D: Cutting vertically through the junction of the lateral and medial parts should be avoided; the nerves to the frontalis muscle course along the outer surface of these layers, so a vertical cut is likely to cross them. The cut should be directed parallel to the outer surface of the skull at the STL so that it separates the frontal pericranium and superficial layer of temporal fascia from the STL while maintaining their continuity. E: The interfascial-subpericranial flap has been reflected forward. F: The temporalis muscle has been detached and can be reflected posteroinferiorly, leaving a narrow cuff of temporal fascia at the STL for closure. Fasc. $=$ fascia; Musc. $=$ muscular. See Figs. $1-3$ for definitions of other abbreviations. 

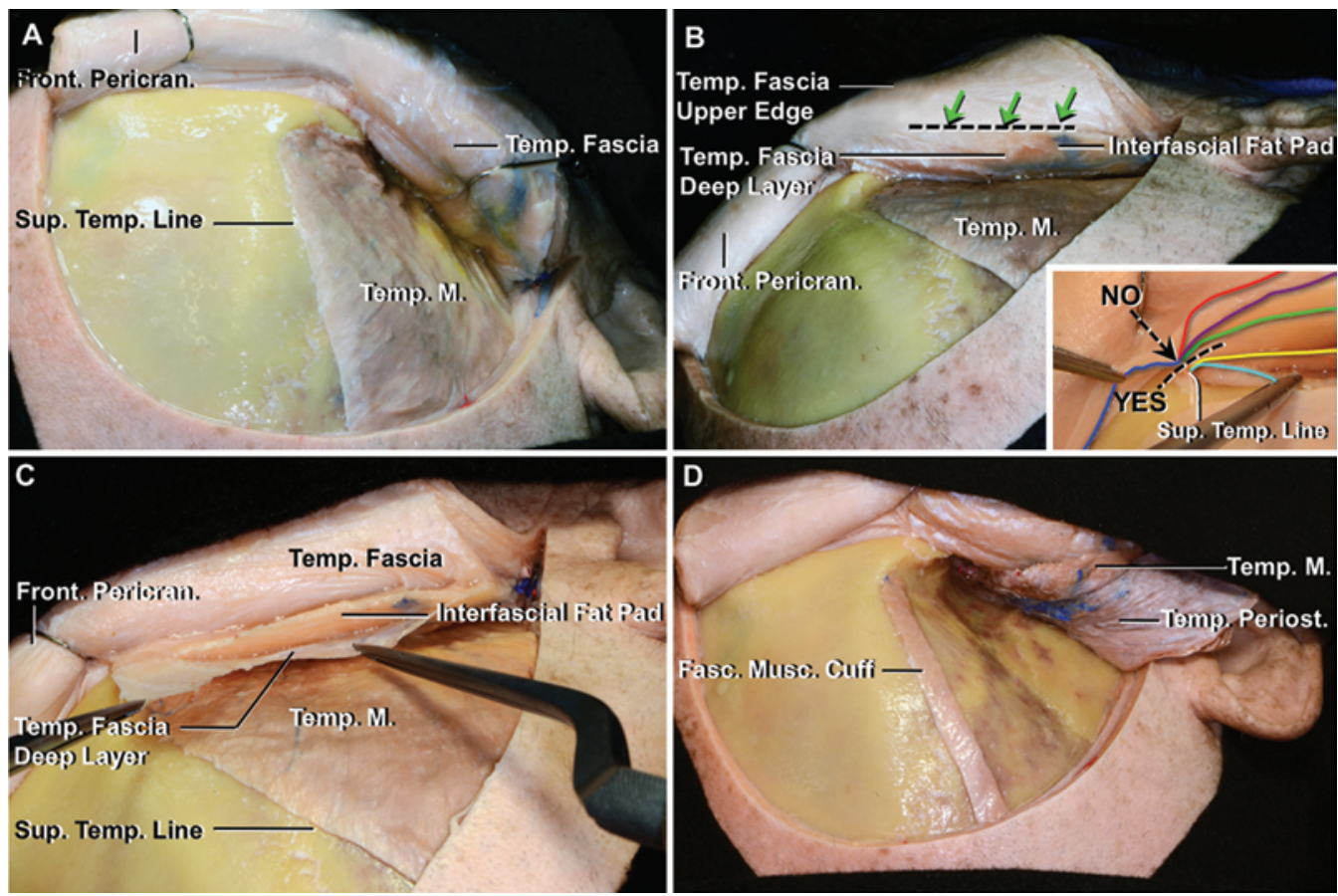

FIG. 5. Photographs of an elevated subfascial-subpericranial flap and underlying structures. A: An elevated subfascial-subpericranial type of flap. The dissection lateral to the STL elevates the deep surface of the temporal fascia from the outer surface of the temporalis muscle in continuity with the frontal pericranium on the medial part of the STL. The temporalis muscle and the periosteum on its deep surface remain attached to the STL. B: The interrupted line shows the upper edge of the interfascial fat pad. The inset shows the incision parallel to the surface of the skull; the incision is used to separate the junction of the temporal fascia lateral to the STL (the superficial layer is edged in red, the deep layer in green, and the interfascial fat pad in purple) and the frontal pericranium (dark blue) medial to the STL while maintaining the continuity of these layers across the STL. C: The deep layer of temporal fascia has been opened from its deep side to expose the upper edge of the interfascial fat pad. There is no need to expose this fat pad in the subfascial-subpericranial approach. D: The temporalis muscle with the periosteum on its deep surface has been elevated and reflected posteriorly. A cuff composed of temporalis muscle and underlying periosteum remains attached along the STL for closure. See Figs. 1-4 for definitions of abbreviations.

of temporal fascia. ${ }^{10}$ Its upper edge is positioned approximately $4 \mathrm{~cm}$ above the lateral orbital rim. 3) The deep fat pad is located between the outer surface of the temporalis muscle and the temporal fascia?

\section{Temporal Branches of the Facial Nerve}

Examination of the course of the temporal branches of the facial nerve in the cadaveric heads revealed the following observations. Immediately superior to the uppermost part of the parotid gland and below the zygomatic arch, the temporal branches of the facial nerve pierced the parotidmasseteric fascia and gave rise to a mean of 3.2 branches (range $2-4$ branches, SD 0.6). Anterior, middle, and posterior branches were common (Figs. 1 and 2). At the level of the zygomatic arch, these branches were located in the loose areolar tissue that, in its first $2 \mathrm{~cm}$ above the arch, was composed of dense fibrofatty tissue bound to the galea with the underlying temporal fascia. At the axial level of the upper edge of the zygomatic arch, the mean distance between the anterior border of the tragus and the most posterior branch of the facial nerve was $15.3 \mathrm{~mm}$ (range $11.0-22.9 \mathrm{~mm}, \mathrm{SD} 3.5 \mathrm{~mm}$ ). The anterior and middle temporal branches then ascended obliquely forward across the STL toward the frontalis and orbicularis muscles. The most posterior temporal branch had a short course, beginning close to the main stem of the superior temporal artery
(STA) and continuing next to the parietal branch of the STA to innervate the auricularis muscle, which is incorporated into the galea above the ear. At the axial level of the lateral canthus of the eye, the branches of the facial nerve that innervated the orbicularis and frontalis muscle were located in the part of the loose areolar layer between the galea and the temporal fascia composed of fibrofatty tissue that loosely binds the galea with the underlying temporal fascia; they had a more anterior ascending course. The nerves to the orbicularis and frontalis muscles commonly originated from the anterior and middle trunks and crossed at a mean distance of $40.4 \mathrm{~mm}$ (range 35.2-45.6 $\mathrm{mm}$, SD $3.3 \mathrm{~mm}$ ) above the lateral canthus of the eye. Those nerves innervating the frontalis muscle then continued their oblique ascending course to pass below the lateral edge of the frontalis muscle to innervate it from its deep side.

Above and medial to the level of the STL, the facial branches coursed in the loose areolar tissue between the galea and frontal pericranium (Fig. 2). The nerve trunks had divided to form a mean of 4.2 tiny branches (range 3-5 branches, SD 0.7) as they crossed the STL (Fig. 2). The most posterior temporal branch to the frontalis muscle that intersected the STL was located a mean distance of $34.9 \mathrm{~mm}$ (range $29.1-40.6 \mathrm{~mm}$, SD $4.4 \mathrm{~mm}$ ) posterosuperior to the lateral canthus of the eye. 
The mean length of the frontalis muscle from the orbital rim to its upper edge was $64.1 \mathrm{~mm}$ (range 57.5-78.5 $\mathrm{mm}$, SD $5.7 \mathrm{~mm}$ ), and the most posterior branch of the facial nerve, passing deep to the lateral border of the frontalis muscle, was located a mean distance of $19.0 \mathrm{~mm}$ (range 14.0-22.5 mm, SD $2.8 \mathrm{~mm}$ ) above the orbital rim (Figs. 1 and 2). Therefore, the branches innervating the frontalis muscle entered predominantly its anterior third and spread upward.

The STA courses in the subcutaneous tissue superficial to the galea and is not normally exposed in the interfascial or subfascial approaches unless the incision crosses the artery or there is a need to expose it for an arterial bypass. The bifurcation of the STA into frontal and parietal branches was located a mean distance of $19.1 \mathrm{~mm}$ (range 5.2-65.5 mm, SD $17.9 \mathrm{~mm}$ ) anterosuperior to the midlevel of the tragus (Figs. 1 and 2) ${ }^{9-12}$ The bifurcation of the STA relative to the zygomatic arch was as follows: 3 branches were inferior, 4 were at the same level, and 3 were superior to it. If the bifurcation of the STA was located above the axial level of the superior orbital rim, the temporal branches of the facial nerve were located predominately anteroinferior to the STA. If the STA bifurcation occurred below this level, one or more branches of the nerve were located superior to or interwoven with the STA.

\section{Discussion}

Prior to the description by Yaşargil et al. ${ }^{16}$ in 1984 of the interfascial technique for preservation of the temporal branches of the facial nerve during pterional craniotomy, frontalis muscle palsy occurred in about $30 \%$ of patients who underwent subgaleal elevation of the scalp flap with separate elevation of the temporalis muscle. ${ }^{3,7,8,11,16}$ This is not surprising considering that the temporal branches to the frontalis muscle run in the loose areolar tissue between the galea and superficial layer of temporal fascia. ${ }^{3}$ The interfascial technique has reduced the incidence of frontalis muscle palsy, although it still occurs. ${ }^{3-5}$

For several reasons, there is still confusion about the best method of dissection to preserve the full course of the nerves to the frontalis muscle, ${ }^{11}$ even when attempting interfascial exposure. One is the inconsistent nomenclature used by different authors to refer to the same structure involved in the procedure. ${ }^{8}$ Krayenbühl et al. ${ }^{11}$ and Davidge et $a{ }^{8}{ }^{8}$ have provided excellent reviews of the different names applied to the layers in the frontotemporal flap, a number of which differ from those commonly used in the neurosurgical literature. Another reason is that the papers describing the technique have focused on the area superficial to the temporalis muscle in the lateral part of the exposure, to which the names "subfascial" and "interfascial" apply, but have failed to emphasize the steps important to maintaining the continuity of the nerves at and medial to the STL. ${ }^{7,11}$

In considering preservation of the nerves to the frontalis muscle, the frontotemporal scalp flap is divided at the STL into lateral and medial parts. The lateral part is associated with the temporalis muscle and its fascia. The goal of the approach to this area is to avoid opening the part of the loose areolar layer between the galea and temporal fascia along which the facial nerve courses. The interfascial and subfascial approaches achieve this goal. The goal of the subpericranial approach (part of the exposure medial to the STL) is to avoid opening the loose areolar layer between the galea and frontal pericranium along which the nerves course. The frontal subpericranial approach achieves this by elevating the frontal pericranium with the scalp flap. In combination, the approaches lateral and medial to the STL are referred to as interfascial-subpericranial and subfascial-subpericranial approaches.

Another important consideration is preserving the integrity of the frontalis muscle, which is embedded in the galeal layer superficial to the frontal pericranium (Fig. 1A). This muscle extends approximately $6.5 \mathrm{~cm}$ superior to the orbital rim. The medial part of the frontotemporal (pterional) incision crosses near the hairline, which is at the upper edge of the frontalis muscle in most cases. A cut across the galea less than $6 \mathrm{~cm}$ above the orbital rim may separate the upper part of the muscle from its lower part and its innervation, which commonly enters the muscle above the orbital rim.

\section{Scalp Flaps}

There are 3 flaps that increase the likelihood that the nerves to the frontalis will be preserved in elevating a frontotemporal flap: the myocutaneous, interfascial-subpericranial, and subfascial-subpericranial flaps.

\section{Myocutaneous Flap}

This flap, in which all the layers of the scalp, including the temporalis muscle and its periosteum laterally, are elevated in continuity with the frontal pericranium medially, virtually eliminates the risk of damage to the nerves to the frontalis muscle. This is at the expense of exposure, however, because the bulky flap with the muscle and all the layers of the scalp attached often blocks visualization along the sphenoid ridge and lower margin of the exposure. ${ }^{16}$ The need to enter the plane between the temporalis muscle and the scalp flap so that the temporalis muscle can be reflected posteriorly away from the pterion adds to the risk of damaging the temporal branches, because these nerves may course through the layers being opened. If, after elevating this flap, it is decided to separate the temporalis muscle from the superficial layers, it is best to avoid entering the loose areolar layer through which the nerves to the frontalis muscle pass. It is best to enter the interfascial or subfascial planes lateral to the STL and subpericranial plane medial to the STL while maintaining the continuity of the temporal fascia and frontal pericranium across the STL.

\section{Interfascial-Subpericranial Flap}

The skin incision begins just anterior to the tragus, extends superiorly, and then curves anteriorly behind the hairline (Fig. 4). The level of the plane of dissection adjacent to the scalp incision is different in the parts medial and lateral to the STL and in the upper and lower parts superficial to the temporalis muscle. In the superior part lateral to the STL, the initial dissection is between the galea and temporal fascia; in the medial part, the dissection extends along the skull deep to the frontal pericranium. 
Approximately $4 \mathrm{~cm}$ above the lateral orbital rim, the interfascial fat pad comes into view between the superficial and deep layers of temporal fascia. At this level, the part of the flap elevation lateral to the STL continues in the interfascial plane deep to the superficial temporal fascia (Fig. 4A-C). Medial to the STL, the dissection continues along the skull deep to the frontal pericranium. The interfascial fat pad is carefully dissected, leaving only the deep layer of the temporal fascia attached to the outer surface of the lower part of the temporalis muscle.

Because the superficial and deep layers of temporal fascia attach to each other and to the frontal pericranium at the STL, it is not possible to fold the interfascial-subpericranial flap forward if these layers remain attached to the STL. Therefore, a carefully tailored cut that separates the attachment of the superficial layer of temporal fascia and the pericranium from the STL is needed so that the frontal pericranium and the superficial layer of temporal fascia, with the nerves on their outer surfaces, can be folded forward with the scalp flap (Fig. 4C and D). This step is not shown in any of the reports on the interfascial technique. A cut directed parallel to the skull surface between the deep and superficial layers of the temporal fascia and deep to the frontal pericranium at their insertion to the STL, while maintaining the continuity between the laterally placed superficial layer of temporal fascia and the medially placed frontal pericranium flap, maintains the continuity of the nerves crossing the STL.

The scalp flap in the interfascial-subpericranial technique includes the superficial layer of temporal fascia in its lateral part and the frontal pericranium in its medial part, which are reflected forward in continuity. Interrupting the continuity of the superficial layer of temporal fascia and frontal pericranium at the STL likely will result in the interruption of the nerves to the frontalis muscle on their outer surface. Finally, the deep periosteal surface of the temporalis muscle is separated from its attachment along the temporal fossa and reflected posteroinferiorly, using careful subperiosteal dissections along its deep surface to preserve its innervation by the deep temporal branches of V3 and its arterial supply from the deep temporal branches of the maxillary artery (Fig. 4E and F).

\section{Subfascial-Subpericranial Flap}

The main difference between the interfascial-subpericranial technique and the subfascial-subpericranial technique presented by Coscarella et al. ${ }^{7}$ is that the superficial and the deep layers of temporal fascia are elevated from the surface of the temporalis muscle in continuity with the frontal pericranium medial to the STL (Fig. 5). There is still a need to separate the insertion of the temporal fascia and frontal pericranium from the STL to turn the flap forward, but this cut should not disrupt the continuity between the temporal fascia and the frontal pericranium (Fig. 5B). We refer to this technique as the subfascial-subpericranial flap to emphasize the importance of the temporal fascia on the lateral portion of the flap, and of the frontal pericranium on its medial side, which are reflected forward in continuity with the scalp flap. One concern with this approach is that separation of the deep temporal fascia from the muscle may damage the surface of the tempora- lis muscle. At trainee courses, it is not uncommon to see some bundles of muscle left adherent to the deep surface of the temporal fascia.

\section{Other Considerations}

Another consideration in this technique is the microscopic terminal filaments innervating the frontalis muscle distal to the point that they cross the STL. The nature of surgery is that it is easier to dissect and preserve structures that can be seen. In pterional craniotomy, the best alternative is to plan the approach so the nerves between the zygomatic arch and the frontalis muscle are not seen and the layers between which they are sandwiched are not opened.

Another potential problem is that confusing the subgaleal fat pad, in which the facial branches course, with the interfascial fat pad, especially in obese patients, can lead to dissection in the plane where the facial nerve branches are located. ${ }^{3,4}$ It has also been suggested that a recurrent twig of the facial nerve innervating the frontalis muscle may run in the interfascial fat pad or temporal neural twigs running in the subgaleal space may lead to damage of the nerves to the frontalis muscle. ${ }^{3,4}$ However, neither of these suggestions has been confirmed in anatomical studies. It seems more likely that the tiny neural branches running in the interfascial fat pad are sensory fibers of the zygomaticotemporal branches arising from V2 rather than motor branches of the facial nerve. ${ }^{10,13}$ Branches of the facial nerve have not been found in the interfascial fat pad.

\section{Conclusions}

The interfascial-subpericranial and subfascial-subpericranial techniques, when accurately completed and with continuity of the temporal fascia, frontal pericranium, and the nerves across the STL maintained, will preserve the innervation of the frontalis muscle.

\section{Acknowledgments}

We deeply appreciate Robin Barry's assistance with the preparation of the illustrations, as well as the editorial assistance of Jessica Striley and Maria Donoso.

\section{References}

1. Abul-Hassan HS, von Drasek Ascher G, Acland RD: Surgical anatomy and blood supply of the fascial layers of the temporal region. Plast Reconstr Surg 77:17-28, 1986

2. Agarwal CA, Mendenhall SD III, Foreman KB, Owsley JQ: The course of the frontal branch of the facial nerve in relation to fascial planes: an anatomic study. Plast Reconstr Surg 125:532-537, 2010

3. Ammirati M, Spallone A, Ma J, Cheatham M, Becker D: An anatomicosurgical study of the temporal branch of the facial nerve. Neurosurgery 33:1038-1044, 1993

4. Ammirati M, Spallone A, Ma J, Cheatham M, Becker D: Preservation of the temporal branch of the facial nerve in pterional-transzygomatic craniotomy. Acta Neurochir (Wien) 128:163-165, 1994

5. Aoki N: Incision of facial nerve branch at aneurysm surgery. J Neurosurg 66:482, 1987 (Letter)

6. Campero A, Socolovsky M, Martins C, Yasuda A, Torino R, Rhoton AL: Facial-zygomatic triangle: a relationship between the extracranial portion of facial nerve and the zygomatic arch. Acta Neurochir (Wien) 150:273-278, 2008 
7. Coscarella E, Vishteh AG, Spetzler RF, Seoane E, Zabramski JM: Subfascial and submuscular methods of temporal muscle dissection and their relationship to the frontal branch of the facial nerve. Technical note. J Neurosurg 92:877-880, 2000

8. Davidge KM, van Furth WR, Agur A, Cusimano M: Naming the soft tissue layers of the temporoparietal region: unifying anatomic terminology across surgical disciplines. Neurosurgery 67 (3 Suppl Operative):ons120-ons130, 2010

9. Kawashima M, Rhoton AL Jr, Tanriover N, Ulm AJ, Yasuda A, Fujii K: Microsurgical anatomy of cerebral revascularization. Part I: anterior circulation. J Neurosurg 102:116-131, 2005

10. Kim S, Matic DB: The anatomy of temporal hollowing: the superficial temporal fat pad. J Craniofac Surg 16:651-654, 2005

11. Krayenbühl N, Isolan GR, Hafez A, Yaşargil MG: The relationship of the fronto-temporal branches of the facial nerve to the fascias of the temporal region: a literature review applied to practical anatomical dissection. Neurosurg Rev 30:8-15, 2007

12. Lei T, Xu DC, Gao JH, Zhong SZ, Chen B, Yang DY, et al: Using the frontal branch of the superficial temporal artery as a landmark for locating the course of the temporal branch of the facial nerve during rhytidectomy: an anatomical study. Plast Reconstr Surg 116:623-630, 2005

13. Martins C, Li X, Rhoton AL Jr: Role of the zygomaticofacial foramen in the orbitozygomatic craniotomy: anatomic report. Neurosurgery 53:168-173, 2003

14. Salas E, Ziyal IM, Bejjani GK, Sekhar LN: Anatomy of the frontotemporal branch of the facial nerve and indications for interfascial dissection. Neurosurgery 43:563-569, 1998
15. Stuzin JM, Wagstrom L, Kawamoto HK, Wolfe SA: Anatomy of the frontal branch of the facial nerve: the significance of the temporal fat pad. Plast Reconstr Surg 83:265-271, 1989

16. Yaşargil MG, Reichman MV, Kubik S: Preservation of the frontotemporal branch of the facial nerve using the interfascial temporalis flap for pterional craniotomy. Technical article. J Neurosurg 67:463-466, 1987

17. Youssef AS, Ahmadian A, Ramos E, Vale F, van Loveren HR: Combined subgaleal/myocutaneous technique for temporalis muscle dissection. J Neurol Surg B Skull Base 73:387-393, 2012

18. Zabramski JM, Kiriș T, Sankhla SK, Cabiol J, Spetzler RF: Orbitozygomatic craniotomy. Technical note. J Neurosurg 89:336-341, 1998

\section{Author Contributions}

Conception and design: all authors. Acquisition of data: all authors. Analysis and interpretation of data: Rhoton, Poblete, Komune, Matsushima. Drafting the article: Rhoton, Poblete. Critically revising the article: Rhoton, Poblete. Reviewed submitted version of manuscript: Rhoton, Poblete. Approved the final version of the manuscript on behalf of all authors: Rhoton. Statistical analysis: Rhoton, Poblete. Administrative/technical/ material support: Rhoton. Study supervision: Rhoton. Contributed to dissections: Jiang.

\section{Correspondence}

Albert L. Rhoton Jr., Department of Neurosurgery, University of Florida, P.O. Box 100265, Gainesville, FL 32610-0265. email: rhoton@neurosurgery.ufl.edu. 